\title{
Urinary neurotransmitter testing: considerations of spot baseline norepinephrine and epinephrine
}

\author{
Marty Hinz' \\ Alvin Stein ${ }^{2}$ \\ Thomas Uncini ${ }^{3}$ \\ 'Clinical Research, NeuroResearch \\ Clinics Inc., Cape Coral, FL, USA; \\ ${ }^{2}$ Stein Orthopedic Associates, \\ Plantation, FL, USA; ${ }^{3}$ DBS \\ Laboratories, Duluth, MN, USA
}

This article was published in the following Dove Press journal:

Open Access Journal of Urology

3 February 20II

Number of times this article has been viewed

Background: The purpose of this paper is to present the results of statistical analysis of spot baseline urinary norepinephrine and epinephrine assays in correlation with spot baseline urinary serotonin and dopamine findings previously published by the authors. Our research indicates a need for physicians and decision-makers to understand the lack of validity of this type of spot baseline monoamine testing when using it in the decision-making process for neurotransmitter deficiency disorders.

Methods: Matched-pairs $t$-tests were performed for a group of subjects for whom spot baseline urinary norepinephrine and epinephrine assays were performed on samples collected on different days then paired by subject.

Results: The reported laboratory test results for urinary serotonin, dopamine, norepinephrine, and epinephrine, obtained on different days from the same subjects, differed significantly and were not reproducible.

Conclusion: Spot baseline monoamine assays, in subjects not suffering from a monoaminesecreting tumor, such as pheochromocytoma or carcinoid syndrome, are of no value in decision-making due to this day-to-day variability and lack of reproducibility. While there have been attempts to integrate spot baseline urinary monoamine assays into treatment of peripheral or central neurotransmitter-associated disease states, diagnosis of neurotransmitter imbalances, and biomarker applications, significant differences in day-to-day reproducibility make this impossible given the known science as it exists today.

Keywords: neurotransmitter testing, epinephrine, norepinephrine, dopamine, serotonin

\section{Introduction}

A previously published paper by the authors of this paper discussed the reproducibility of spot baseline urinary serotonin and dopamine assays. ${ }^{1}$ This companion paper discusses the reproducibility of spot baseline urinary norepinephrine and epinephrine assays, and explores the feasibility and validity of using spot urinary norepinephrine or epinephrine assays in subjects not suffering from a monoamine-secreting tumor as a basis for decision-making. The paper then correlates the novel spot baseline norepinephrine and epinephrine findings reported here with our earlier reports relating to spot baseline urinary serotonin and dopamine.

Urinary neurotransmitter testing samples can be generated in several ways. "Spot urine" is a single urine sample obtained at a specific time. ${ }^{1}$ A 24-hour urine sample is a collection of all urine excreted in a defined time period, and is used when the total daily excretion of a substance by the kidneys into the urine is to be studied. One application of the 24-hour urine test is in the diagnosis of monoamine-secreting tumors.
Correspondence: Marty Hinz 1008 Dolphin Dr, Cape Coral, FL 33904, USA

$\mathrm{Tel}+\mathrm{I} 2186262220$

Fax +I 2186261638

Emailmarty@hinzmd.com
Open Access Journal of Urology 201 1:3 19-24

(C) 20II Hinz et al, publisher and licensee Dove Medical Press Ltd.This is an Open Access article

Dovepress which permits unrestricted noncommercial use, provided the original work is properly cited. 
Collection of a 24-hour urine sample is burdensome, and requires the subject to carry sample collection materials during all daily activities. ${ }^{2,14}$

Urinary monoamines exist in two states, ie, "the endogenous state", found when no amino acid precursors of the monoamines are being administered, and "the competitive inhibition state", found when significant amounts of both serotonin and dopamine amino acid precursors are being administered simultaneously. Obtaining urine samples in the endogenous state is known as "baseline testing". ${ }^{3}$ The focus of this paper is spot urine measurements obtained in the endogenous state, which is also known as "baseline urinary neurotransmitter testing". Spot baseline urinary neurotransmitter testing samples obtained in the endogenous state are of no value in patients not suffering from a monoamine-secreting tumor, such as pheochromocytoma or carcinoid syndrome, due to a lack of reproducibility of the testing involved. ${ }^{1}$ Previously published peer-reviewed literature has established the validity and utility of OCT interpretation of monoamine assays in the competitive inhibition state when performed under proper conditions. ${ }^{1,3-5}$

\section{Materials and methods}

Results of statistical analysis of spot baseline urinary neurotransmitter testing of serotonin and dopamine assays have been discussed and published previously by the authors of this paper. ${ }^{1}$ Novel statistical results of spot baseline urinary neurotransmitter testing of norepinephrine and epinephrine assays from a database accumulated by two of the authors of this paper are reported here. Urinary norepinephrine and epinephrine samples obtained on different days from the same subject were statistically analyzed using a matchedpairs $t$-test. A $P$ value $\leq 0.05$ was considered to reveal a significant difference between groupings. JMP (SAS Institute, Cary, NC) software was used to perform the statistical analysis.

Processing, management, and assay of the urine samples collected for this study were as follows. Urine samples were collected six hours prior to bedtime, with $4 \mathrm{pm}$ being the most frequent collection time point. The samples were stabilized in $6 \mathrm{~N} \mathrm{HCl}$ to preserve urinary dopamine and urinary serotonin. Samples were shipped to DBS Laboratories, Duluth, MN. Urinary norepinephrine and dopamine were assayed utilizing commercially available radioimmunoassay kits (3 CAT RIA IB88501 and IB89527; Immuno Biological Laboratories Inc, Minneapolis, MN). DBS Laboratories is accredited as a high complexity laboratory by Clinical Laboratory Improvement Amendments to perform these assays.

\section{Results}

In order for laboratory testing to be valid it needs to be reproducible. The following is a discussion of the statistical reproducibility of spot baseline urinary neurotransmitter testing of norepinephrine and epinephrine performed on a group of subjects in whom two urine samples were obtained on different days. The matched-pairs $t$-test was used to evaluate these spot baseline samples. To complete the serotonin and catecholamine discussion, previously published data by the authors relating to spot baseline urinary neurotransmitter testing of serotonin and dopamine is included, because norepinephrine and epinephrine production and balance are related to balanced levels of serotonin and dopamine.

\section{Spot baseline norepinephrine matched-pairs t-test}

The following norepinephrine data are novel. The laboratory values are reported in $\mu \mathrm{g}$ of norepinephrine per $\mathrm{g}$ of creatinine. From a matched-pairs group of $\mathrm{n}=54$, the mean and standard deviation (SD) for both spot baseline norepinephrine urinary assay groups was determined. For Group 1, the mean norepinephrine value was found to be $64.66( \pm 148.98)$. For Group 2 (spot baseline norepinephrine testing performed on a different day after the first assay), the mean norepinephrine value was found to be $42.01( \pm 173.39)$. All data greater than the value found in calculating the sum of two SDs plus the mean were removed from consideration, revealing a group of $n=44$. This matched-pair values group was then analyzed using the matched-pairs $t$-test, revealing a $P$ value of 0.0399 . These findings indicate that spot baseline urinary norepinephrine levels do differ in a statistically significant manner when spot baseline assays are performed on different days from the same subject. This supports the assertion that spot urinary norepinephrine values are not uniform or reproducible from day to day. The epinephrine group $(n=44)$ comprised 21 females aged $48.22( \pm 13.34)$ years and 23 males aged $46.31( \pm 14.63)$ years.

\section{Spot baseline epinephrine matched-pairs $t$-test}

The following epinephrine data are also novel. The laboratory values are reported in $\mu \mathrm{g}$ of epinephrine per $g$ of creatinine. From a matched-pairs group of $\mathrm{n}=135$, the mean and the SD for both spot baseline epinephrine urinary assay groups was determined. For Group 1, the mean epinephrine value was found to be $6.55( \pm 5.5)$. For Group 2 (spot baseline testing performed on a different day after the first assay), the mean epinephrine value was found to be 10.4 ( \pm 14.12$)$. 
All data greater than the value found in calculating the sum of two SDs plus the mean were removed from consideration, leaving a group of $n=122$. This matched-pair values group was then analyzed using the matched pairs $t$-test, revealing a $P$ value of $<0.0001$. These findings indicate that spot baseline urinary epinephrine levels do differ in a statistically significant manner when spot baseline assays are performed on different days from the same subject. This supports the assertion that spot urinary epinephrine values are not uniform or reproducible from day to day. The epinephrine group $(n=122)$ comprised 63 females aged $59.09( \pm 11.87)$ years and 59 males aged $45.89( \pm 18.72)$ years.

\section{Spot baseline serotonin matched-pairs $t$-test}

A 2010 peer-reviewed paper by the authors presented results of a novel spot serotonin matched-pairs $t$-test $(n=134)$. Spot baseline-baseline grouping of urinary serotonin samples obtained on different days from the same patient revealed a $P$ value of 0.0080 . This indicates that spot baseline urinary serotonin levels differ in a statistically significant manner when they are performed on different days from the same subject. This supports the assertion that spot urinary serotonin values are not uniform or reproducible from day to day. ${ }^{1}$

\section{Spot baseline dopamine matched-pairs $t$-test}

A 2010 peer-reviewed paper by the authors of this paper presented results of a novel spot dopamine matched-pairs $t$-test $(\mathrm{n}=138)$. Spot baseline-baseline grouping of urinary dopamine samples obtained on different days from the same patient revealed a $P$ value of 0.0049 . This indicates that spot baseline urinary dopamine levels differ in a statistically significant manner when they are performed on different days from the same subject. This supports the assertion that spot urinary dopamine values are not uniform or reproducible from day to day. ${ }^{1}$

Results of the four matched-pairs $t$-tests shown in Table 1 reveal that there are significant differences between spot baseline urinary neurotransmitter testing performed on different days from the same subject for all four monoamines under scrutiny.

Simply asserting that testing differs significantly and is not reproducible from day to day in the same subject may not have the impact of reviewing the data used for the statistical analysis. The data in the accompanying tables illustrate that the urinary neurotransmitter testing results are not
Table I Matched-pairs $t$-test values. A $P$ value $<0.05$ indicates that a significant difference between the test I grouping and test 2 grouping exists on different days in the same individual. Spot baseline monoamine assays are not uniform and reproducible from day to day in the same subject, and therefore the testing is not reproducible or valid

\begin{tabular}{lll}
\hline & $\mathbf{n}$ & $\boldsymbol{P}$ value \\
\hline Norepinephrine & 44 & 0.0399 \\
Epinephrine & 122 & $<0.0001$ \\
Serotonin & 134 & 0.0080 \\
Dopamine & 138 & 0.0049 \\
\hline
\end{tabular}

reproducible from day to day, and that spot baseline urinary neurotransmitter testing is not a valid foundation for medical decision-making. Tables 2-5 contain the paired results of 160 spot baseline urinary neurotransmitter tests. All values are reported in $\mu \mathrm{g}$ of monoamine per $g$ of creatinine.

The urine samples analyzed were collected approximately six hours prior to bedtime, with $4 \mathrm{pm}$ being the most common time of collection. A review of all samples collected at other times of the day revealed results that were similar to the aforementioned findings. Spot baseline urinary monoamine samples differed significantly from day to day in the same subject, regardless of the time collected, and were not reproducible.

\section{Discussion}

In the scientific world, there are two highly polarized views regarding the validity of spot baseline urinary neurotransmitter testing. One view advocates that baseline urinary neurotransmitter testing has no value in patients not suffering from a

Tables 2a, b Serial spot baseline-baseline norepinephrine assays from the same subject. Some of the norepinephrine data used to determine the norepinephrine matched-pairs $t$-test values found in Table I. Comparison of norepinephrine I with norepinephrine 2 from the same subject (by row) illustrates the lack of test reproducibility. The number of days column is the number of days between urinary sample collection dates

\begin{tabular}{|c|c|c|c|c|c|}
\hline \multicolumn{3}{|c|}{ a) Sort: High-low by NE-I } & \multicolumn{3}{|c|}{ b) Sort: High-low by NE-2 } \\
\hline Days (n) & NE-I & NE-2 & Days (n) & NE-I & NE-2 \\
\hline 217 & 595.42 & 270.20 & 272 & 145.46 & 861.92 \\
\hline 58 & 479.59 & 8.50 & 225 & 7.67 & 581.60 \\
\hline 28 & 416.86 & 132.37 & 32 & 386.01 & 540.17 \\
\hline 41 & 399.75 & 49.38 & 79 & 151.44 & 482.38 \\
\hline 32 & 386.01 & 540.17 & 217 & 595.42 & 270.20 \\
\hline 19 & 381.86 & 10.62 & 29 & 232.14 & 261.01 \\
\hline 42 & 357.80 & 61.73 & 189 & 132.09 & 233.98 \\
\hline 41 & 301.00 & 203.70 & 41 & 301.00 & 203.70 \\
\hline 50 & 268.04 & 31.36 & 28 & 0.97 & 195.92 \\
\hline 29 & 232.14 & 261.01 & 64 & 214.24 & 186.00 \\
\hline
\end{tabular}

Abbreviation: NE, norepinephrine. 
Tables 3a, b Serial spot baseline-baseline epinephrine assays from the same subject, including epinephrine data used to determine the epinephrine matched-pairs t-test values found in Table I. Comparison of EPI-I with EPI-2 from the same subject (by row) illustrates lack of test reproducibility. The number of days column is the number of days between urinary sample collection dates

\begin{tabular}{|c|c|c|c|c|c|}
\hline \multicolumn{3}{|c|}{ a) Sort: High-low by EPI-I } & \multicolumn{3}{|c|}{ b) Sort: High-low by EPI-2 } \\
\hline Days (n) & EPI-I & EPI-2 & Days (n) & EPI-I & EPI-2 \\
\hline 43 & 36.06 & 3.90 & 77 & 8.98 & 29.09 \\
\hline 22 & 24.83 & 9.58 & 272 & 13.09 & 16.37 \\
\hline 364 & 22.81 & 11.99 & 104 & 8.43 & 15.34 \\
\hline 27 & 21.37 & 3.22 & 35 & 8.62 & $|5.0|$ \\
\hline 46 & 20.44 & 14.76 & 42 & 14.80 & 14.99 \\
\hline 49 & 18.80 & 6.69 & 46 & 20.43 & 14.76 \\
\hline 380 & 18.59 & 13.83 & 98 & 6.39 & 14.10 \\
\hline 185 & 16.49 & 4.87 & 380 & 18.59 & 13.83 \\
\hline 42 & 14.80 & 8.43 & 225 & 6.16 & 13.42 \\
\hline 41 & 12.86 & 6.57 & 22 & 24.83 & 13.02 \\
\hline
\end{tabular}

Abbreviation: EPI, epinephrine.

monoamine-secreting tumor. ${ }^{1,3-5}$ The other view advocates that it is very beneficial, and that it has numerous applications in medical decision-making, including diagnostic, therapeutic, and biomarker applications. ${ }^{6-12}$ The purpose of this writing is to educate medical practitioners regarding the selection of laboratory testing for neurotransmitter diseases so that they do not use invalid testing methods.

The science supporting the view of the authors is as follows. It is a well-known fact that norepinephrine, epinephrine, serotonin, and dopamine do not cross the blood-brain barrier. These monoamines are filtered at the glomerulus and are then metabolized by the kidneys. Significant amounts of these monoamines filtered at the glomerulus do not reach the final urine. Monoamines found in the urine of patients not suffering from a monoamine-secreting tumor are primarily synthesized by structures in the kidneys. ${ }^{1,3-5,13}$ Spot baseline testing lacks reproducibility and is of no value in patients not suffering from a monoamine-secreting tumor. ${ }^{1}$

Those who claim that spot baseline urinary neurotransmitter testing is valid assert that monoamines cross the blood-brain barrier, are filtered at the glomerulus, and simply excreted into the urine without further renal involvement. They conclude that spot baseline urinary neurotransmitter testing is a valid assay for peripheral and central nervous system neurotransmitter levels. ${ }^{6-12}$

Spot baseline urinary neurotransmitter testing of norepinephrine, epinephrine, serotonin, and dopamine is not reproducible from day to day in the same subject; therefore, this type of testing is not valid. An infinite number of assays performed on an infinite number of days would generate an infinite number of differing test results. ${ }^{1}$ The following are true, based on the statistics put forth in this paper and the lack of reproducibility as demonstrated in this writing:

- Spot urinary neurotransmitter testing is not a reliable assay for peripheral or central nervous system function; the majority of serotonin and catecholamine molecules found in the urine of patients not suffering from a monoamine-secreting tumor have never been in the peripheral or central nervous system, having been synthesized by renal structures

- Spot urinary neurotransmitter testing does not correlate with monoamine neurotransmitter-related disease states in patients not suffering from a monoamine-secreting tumor

- Spot urinary neurotransmitter testing, due to lack of reproducibility, cannot assist the health care practitioner in making informed decisions regarding the choice

Tables 4a, b Serial spot baseline-baseline serotonin assays from the same subject, including some of the serotonin data used to determine the serotonin matched-pairs $t$-test values found in Table I, from a previously published paper by the authors.' Comparison of serotonin I with serotonin 2 from the same subject (by row) vividly illustrates lack of testing reproducibility. The number of days column is the number of days between urinary sample collection dates

\begin{tabular}{|c|c|c|c|c|c|}
\hline \multicolumn{3}{|c|}{ a) Sort: High-low by Serotonin I } & \multicolumn{3}{|c|}{ b) Sort: High-low by Serotonin 2} \\
\hline Days & Serotonin I & Serotonin 2 & Days & Serotonin I & Serotonin 2 \\
\hline 42 & 9885.64 & 179.65 & 272 & 307.07 & 6004.24 \\
\hline 28 & 5178.39 & 415.45 & 79 & 1159.95 & 5194.81 \\
\hline 32 & 3309.76 & 1191.05 & 41 & 2451.00 & 4049.95 \\
\hline 41 & 2451.00 & 4049.95 & 41 & 96.77 & 3655.97 \\
\hline 98 & 2157.10 & 368.47 & 103 & 9885.65 & 3246.75 \\
\hline 42 & 1569.16 & 432.35 & 217 & 828.22 & 2275.38 \\
\hline 79 & 1159.95 & 5194.81 & 204 & 276.97 & 2183.79 \\
\hline 29 & 1005.58 & 851.43 & 47 & 227.30 & 2000.00 \\
\hline 217 & 828.22 & 2275.38 & 383 & 60.32 & 1996.24 \\
\hline 19 & 763.47 & 31.14 & 32 & 3309.76 & II9I.05 \\
\hline
\end{tabular}


Tables 5a, b Serial spot baseline-baseline dopamine assays from the same subject, including dopamine data from a previous study used to determine the dopamine matched-pairs $t$-test values found in Table I.' Comparison of dopamine I with dopamine 2 from the same subject (by row) illustrates the lack of test reproducibility. The number of days column is the number of days between urinary sample collection dates

\begin{tabular}{|c|c|c|c|c|c|}
\hline \multicolumn{3}{|c|}{ a) Sort: High-low by dopamine I } & \multicolumn{3}{|c|}{ b) Sort: High-low by dopamine 2} \\
\hline Days (n) & Dopamine I & Dopamine 2 & Days (n) & Dopamine I & Dopamine 2 \\
\hline 46 & 7854.32 & 1884.93 & 41 & 1129.58 & 2891.23 \\
\hline 41 & 1129.58 & 2891.23 & 98 & 300.37 & 2623.79 \\
\hline 204 & 1034.63 & 71.76 & 6 & $|38.8|$ & 2504.14 \\
\hline 28 & 785.00 & 181 & 103 & 164.50 & 2109.03 \\
\hline 77 & 652.35 & 1288.47 & 46 & 7854.32 & 1884.93 \\
\hline 27 & 498.23 & 68.80 & 28 & 785.00 & 1806.00 \\
\hline 58 & 419.82 & 88.41 & 77 & 652.35 & 1288.48 \\
\hline 168 & 405.20 & $|80.5|$ & 314 & 197.72 & 1220.54 \\
\hline 28 & 387.64 & 169.78 & 47 & 785.00 & 853.00 \\
\hline 29 & 372.51 & 208.49 & 383 & 289.88 & 430.71 \\
\hline
\end{tabular}

of amino acids, or the dosing value for intervention with a disease state associated with monoamine neurotransmitters

- Spot urinary neurotransmitter testing, due to lack of reproducibility, does not have a place in clinical practice for identifying biomarkers of peripheral or central nervous system function and disease states

- Spot urinary neurotransmitter testing cannot determine monoamine imbalances that exist in subjects because the results are not reproducible

- Spot baseline monoamine assays cannot serve as a predictor of expected efficacy once amino acid precursors are started due to lack of reproducibility.

There is evidence that urinary monoamines, such as norepinephrine reported on 24-hour urine samples, may be elevated in a specific group of patients with depression. ${ }^{15}$ However, these are group findings, and do not necessarily translate to individual testing validity on spot testing due to the lack of reproducibility of the test from day to day in the same subject.

\section{Conclusion}

This research underscores the fallacy of the attempt to use spot baseline urinary neurotransmitter testing as a potential biomarker in the treatment of patients with presumed monoamine neurotransmitter-related diseases who are not suffering from a monoamine-secreting tumor. Levels of urinary norepinephrine, epinephrine, serotonin, and dopamine, found in the urine on spot baseline testing, differ significantly from day to day in the same subject. Results are not reproducible, so spot baseline urinary neurotransmitter testing in the endogenous state in subjects not suffering from a monoamine-secreting tumor is of no clinical value. Health care practitioners need to understand this difference when selecting a form of testing for their patients. It is hoped that this writing will spark interest and scrutiny of the topic, leading to advancement of the relevant science.

\section{Disclosure}

The authors report no conflicts of interest in this work.

\section{References}

1. Hinz M, Stein A, Trachte G, Uncini T. Neurotransmitter testing of the urine, a comprehensive analysis. Open Access Journal of Urology. 2010;2:177-183.

2. Smythe G, Edwards G, Graham P, Lazarus L. Biochemical diagnosis of pheochromocytoma by simultaneous measurement of urinary excretion of epinephrine and norepinephrine. Clin Chem. 1992;38: 486-492.

3. Hinz M, Stein A, Uncini T. The dual-gate lumen model of renal monoamine transport. Neuropsychiatr Dis Treat. 2010;6:387-392.

4. Hinz M. Depression. In: Kohlstadt I, editor. Food and Nutrients in Disease Management. Boca Raton, FL: CRC Press; 2009.

5. Hinz M, Stein A, Uncini T. Amino acid responsive Crohn's disease. Clinical and Experimental Gastroenterology. 2010;3:171-177.

6. Alts J, Alts D, Bull M. Urinary Neurotransmitter Testing: Myths and Misconceptions. Osceola, WI: NeuroScience Inc; 2007.

7. Watkins R. Validity of urinary neurotransmitter testing with clinical applications of CSM (Communication System Management) model. Asheville, NC: Sanesco International; 2009. Available at: http://www.neurolaboratory.net/1ab/neurolab\%20pdf.\%20 files $/ 2009 \% 20$ Urinary $\% 20$ NT\%20White\%20Paper.pdf. Accessed November 24, 2010.

8. Theirl S. Clinical relevance of neurotransmitter testing. The Original Internist. December 2009. Available at: http://www.clintpublication. com/documents/Dec_OI_2009.pdf. Accessed November 24, 2010.

9. Sanesco. Neurolab baseline sample report. Available at: http://sanesco. net/images/files/resourcelibrary/baseline_sample_report.pdf. Accessed November 24, 2010.

10. Neuroscience. Assessing nutritional imbalances. Available at: https:// www.neurorelief.com/index.php?option=com_content\&task=view\&id= 131\&Itemid=48. Accessed November 24, 2010. 
11. Kellermann G, Bull M, Ailts J, et al. Understanding diurnal variation. Technical Bulletin Issue 4. Osceola, WI: NeuroScience Inc; January 9, 2004. Available at: https://www.neurorelief.com/index. php?option=com_content\&task=view\&id=224\&Itemid=48. Accessed November 24, 2010.

12. Marc D, Ailts J, Ailts-Campeau D, et al. Neurotransmitters excreted in the urine as biomarkers of nervous system activity: validity and clinical applicability. Neurosci Biobehav Rev. 2011;35:635-644.
13. Trachte G, Uncini T, Hinz M. Both stimulatory and inhibitory effects of dietary 5-hydroxytryptophan and tyrosine are found on urinary excretion of serotonin and dopamine in a large human population. Neuropsychiatr Dis Treat. 2009;5:227-235.

14. Hughes J, Watkins L, Blumenthal J, Kuhn C, Sherwood A. Depression and anxiety symptoms are related to increased 24-hour urinary norepinephrine excretion among healthy middle-aged women. $J$ Psychosom Res. 2004;57:353-358.

\section{Publish your work in this journal}

The Open Access Journal of Urology is an international, peer-reviewed, open access journal publishing original research, reports, editorials, reviews and commentaries on all aspects of adult and pediatric urology in the clinic and laboratory including the following topics: Pathology, pathophysiology of urological disease; Investigation and treatment of urological disease; Pharmacology of drugs used for the treatment of urological disease. The manuscript management system is completely online and includes a very quick and fair peer-review system, which is all easy to use. Visit http://www.dovepress.com/testimonials.php to read real quotes from published authors.

Submit your manuscript here: http://www.dovepress.com/open-access-journal-of-urology-journal 